\title{
A Survey of Koreans on Sleep Habits and Sleeping Symptoms Relating to Pillow Comfort and Support
}

\author{
Juhyun Son, Sungwook Jung, Haseung Song, Jihee Kim, Seonghwan Bang and Sangwoo Bahn * \\ Department of Industrial and Management Systems Engineering, Kyung Hee University, 17104 Yongin, Korea; \\ 12thswngus@naver.com (J.S.); jungsw06310@gmail.com (S.J.); hon1415@gmail.com (H.S.); ji_hee@khu.ac.kr (J.K.); \\ bsh7678@gmail.com (S.B.) \\ * Correspondence: panlot@gmail.com; Tel.: +82-31-201-3654; Fax: +82-31-203-4004
}

Received: 13 October 2019; Accepted: 29 December 2019; Published: 1 January 2020

\begin{abstract}
The number of people who complain of sleep disturbances is steadily increasing. An understanding of sleep-related factors is required to address sleep problems. This survey study investigated the sleep habits and sleeping symptoms relating to the comfort and support characteristics of pillows and the relationship between sleep quality and pillow design factors. The study utilized data from 332 participating Korean adults aged 20-76 years (mean age \pm SD: males, $40.4 \pm 15.2$; females, $42.9 \pm 15.4$ ). We developed a questionnaire that evaluated sleep habits (sleep duration, bedtime, wake-up time and sleeping position); sleeping symptoms (snoring or coughing, breathing and sleepiness during waking hours) based on the Korean version of the Pittsburgh Sleep Quality Index (PSQI-K) questionnaire; and pillow-related factors (support, comfort, fatigue, height and shape) from existing pillow studies. The average sleep duration was $6.8 \mathrm{~h}$, with more than half $(52 \%)$ of participants sleeping in the supine position. The overall score for sleep quality was considered poor (4.84 points on a seven-point Likert scale), with some degree of sleepiness during waking hours (4.4 points on a seven-point Likert scale). Females went to bed earlier than males and were more likely to sleep in the lateral position compared to males. The number of toss and turn or waking events during sleep increased with age, and older individuals went to sleep earlier and woke up earlier. Among the symptoms of fatigue, pain, discomfort with changing position, snoring, coughing and breathing discomfort, participants reported their highest levels of discomfort due to sleepiness after waking, and they experienced the least head pain. Participants who used a regular-type pillow had poorer satisfaction on multiple comfort and support factors (support, comfort, height suitability, shape suitability) compared with those who used a functional-type pillow. Less head fatigue, less neck fatigue and less shoulder pain had significant effects on sleep quality. To reduce neck fatigue and shoulder pain, designers should consider the height for neck support in the lateral position. To reduce neck fatigue, it is desirable to use materials like latex or memory foam that provide neck support, which can improve sleep quality. The findings of this study contribute to a better understanding of sleep habits and characteristics of pillow comfort and provide practical guidelines for better pillow designs.
\end{abstract}

Keywords: sleep quality; sleeping position; pillow; pillow design; comfort

\section{Introduction}

Sleep accounts for one-third of the human lifespan [1] and is an important activity of everyday life that allows humans to recover physically and psychologically in preparation for the next day [2,3]. The importance of sleep is increasingly emphasized in the public sphere, and the proportion of people complaining of sleep disorders is reportedly increasing [4]. Low-quality sleep is related to less sleep, pain during sleep and breathing problems [5-7]. 
Previous studies have investigated sleep duration, bedtime and wake-up time of Koreans [8-16]. As interest in sleep has increased, so has interest in pillows for restful sleep. Various pillow types have been released into the market with different sizes, heights and materials. The use of proper sleeping accessories is an important way to improve sleep quality. For example, a pillow supports the head during sleep, whereas improper pillow support can cause neck stiffness, physical fatigue and pain, which can reduce sleep quality. Therefore, it is necessary to have a general understanding of the factors that can affect the quality of sleep, such as sleep habits and sleeping symptoms associated with pillow comfort and support.

Previous studies have been conducted on pillow design elements (materials, shape, contour), and pain caused by various pillow types [17-27]. Gordon and Grimmer-Somers [21] reported that latex materials can reduce headache and shoulder pain. In patients with chronic neck pain, a pillow that maintained the correct cervical curve of the neck could reduce neck pain and headaches [23]. In a study of pillow design elements, Kushida et al. [24] reported that cervical pillows decreased abnormal breathing events per hour during sleep in patients with sleep apnea. Cai and Chen [25] analyzed participant's actual sleeping positions and suggested that a pillow with a high side height could improve sleep quality. Ren et al. [26] studied the importance of proper pillow height; as the height of a pillow increased, the average and highest pressure on the head and cervical spine also increased. Liu et al. [27] proposed a new pillow design with a proper combination of head, neck and shoulder support to improve sleep quality.

However, there are many considerations that have not been addressed in previous studies. Few studies have analyzed the relationship between pillow-related factors and sleep habits. In particular, previous studies did not sufficiently consider the different pillow design factors on sleeping symptoms. Previous research has been conducted by selecting a specific pillow and testing its overall shape, often by comparing a regular pillow with a cervical pillow. Shields et al. [17] claimed that there was insufficient evidence that cervical pillows reduced chronic neck pain compared with regular pillows. Therefore, it is necessary to examine pillow design factors, such as shape and materials, to understand how they impact sleep and sleeping symptoms.

The purpose of this study is to investigate the sleep habits and sleeping symptoms relating to the comfort and support characteristics of pillows through a survey in Korean adults, and to analyze any differences by age, sex, sleeping position or pillow type.

\section{Methods}

\subsection{Study Design}

Survey data for this study, using convenience sampling, were collected online and offline for four months (from 1 August to 30 November 2017) after deliberation by the Institutional Review Board of Kyung Hee University (KHSIRB-17-023). Most of the participants lived in a metropolitan area in Korea and comprised students studying in Kyung Hee University (most in their 20s), office workers and housewives (other ages). Only healthy participants were included in the study, as per a questionnaire (aged 20 to 76 years, Body Mass Index (BMI) 17.1 to $40.6 \mathrm{~kg} \cdot \mathrm{m}^{-2}$ ). The total number of participants was 332, and six people were excluded from the analysis because they did not complete the questionnaire or did not respond to the pillow material-type questions. Participants were asked to respond to a questionnaire (Table 1) that consisted of 32 questions designed to assess the sleep habits, sleeping symptoms, comfort and support characteristics of pillows, as well as perceptions of pillow suitability. We developed the questions about sleep habits (average sleep latency, bedtime, wake-up time, sleep duration, number of tosses and turns or waking events and sleeping position during the preceding month) and symptoms (snoring or coughing, breathing and sleepiness during waking hours) based on the Korean version of the Pittsburgh Sleep Quality Index (PSQI-K) and questions about pillow-related factors (support, comfort, fatigue, height and shape) from existing pillow studies [17-27]. 
Table 1. Questionnaire to assess sleep habits, sleeping symptoms and the comfort and support characteristics of pillows.

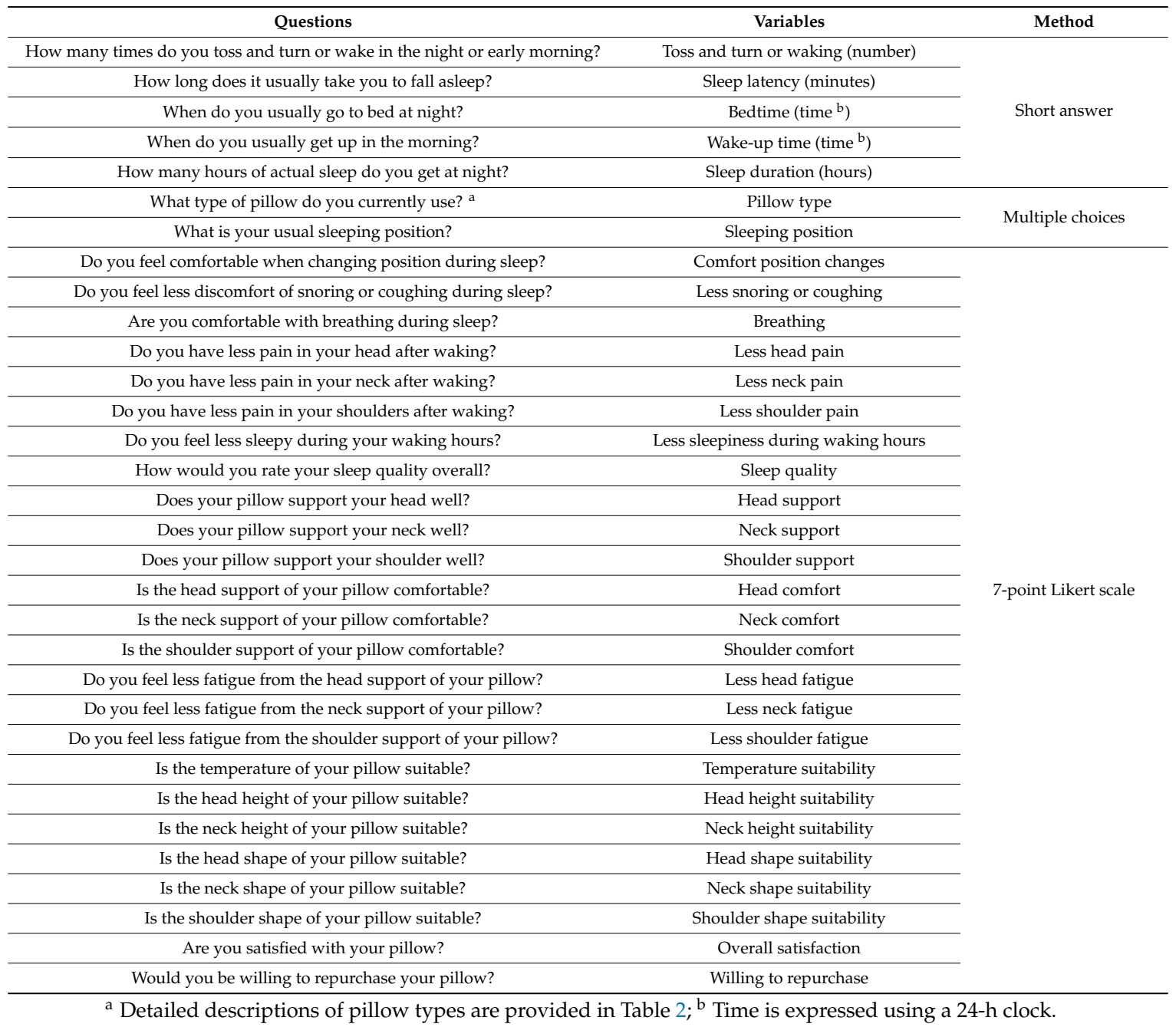

\subsection{Sleep Habits and Sleeping Symptoms}

To develop survey questions for this study, the factors related to sleep and representative methods were examined to evaluate subjective sleep habits and sleeping symptoms relating to the comfort and support characteristics of pillows. Common questions were extracted to understand the sleep habits of healthy adults. Pilcher et al. [5] reported that indicators that affected sleep included quantitative factors such as sleep duration, bedtime, wake-up time and number of waking events during sleep, as well as qualitative factors such as self-reported quality of sleep or satisfaction with sleep. Common factors assessed by researchers included sleep duration, sleep quality, bedtime and sleep latency [28-32]. Methods for subjectively evaluating sleep quality were used, including the Verran and Snyder-Halpern Sleep Scale [28], the Pittsburgh Sleep Quality Index (PSQI) [33] and the Karolinska Sleep Diary [34]. Among those, the PSQI is the most commonly used measure [35-37], and it measures sleep quality and sleep disturbance during the preceding month. Our sleep habit questions were based on the PSQI-K which has been tested for its reliability and validity [38]. In addition, some questions about subjective assessment were added using a Likert scale (see Table 1 for the entire questionnaire). The Likert scale for the overall sleep quality was answered using the following criteria: for sleep quality, with 1 being low sleep quality and 7 high sleep quality; for sleepiness during the day, with 1 being discomfort and 7 comfort; and for the number and degree of severity of sleep-related symptoms with $1=$ strong negative, $2=$ negative, $3=$ somewhat negative, $4=$ neutral, $5=$ somewhat positive, $6=$ positive and 
7 = strong positive. The question of sleeping position was largely divided into supine and lateral positions. The supine position was defined as "Mainly sleep in a supine position" and "Mainly sleep in a supine position but also sleep in a lateral position". The lateral position was defined as "Mainly sleep in a lateral position" and "Mainly sleep in a lateral position but also sleep in a supine position".

Table 2. Classification and definition of pillow types.

\begin{tabular}{|c|c|c|}
\hline Pillow Type & Definition & Example \\
\hline Regular-type pillow & - Typical oval or rectangular shape & \\
\hline Contour-type pillow & - Different heights for head and neck support & \\
\hline Peanut-shaped pillow & $\begin{array}{l}\text { - Different heights for head and neck support } \\
\text { - U-shaped to surround the neck and shoulders }\end{array}$ & \\
\hline Functional A-type pillow & $\begin{array}{l}\text { - Different heights for head and neck support } \\
\text { - Neck supported by a neck support }\end{array}$ & \\
\hline Functional B-type pillow & $\begin{array}{l}\text { - Different heights for head and neck support } \\
\text { - Neck surrounded by a neck support }\end{array}$ & \\
\hline Other & - Wooden pillow, etc. & \\
\hline
\end{tabular}

\subsection{Pillow Types}

Market research and a related literature review were conducted to develop questions about the comfort and support characteristics associated with pillow use. Previous studies about pillows were systematically reviewed and information about more than 200 commercially available pillows was collected and classified into six types according to shape and contour (Table 2). According to the studies of Yokura et al. [39] and Hur and Yang [22], design factors of pillow type such as height shape, and subjective factors of pillow comfort and fatigue could affect pillow usability. In this study, we selected support, height and shape as pillow design factors. Since a pillow affects the head, neck and shoulder comfort, questions directed to these body parts in terms of comfort, fatigue, pain and overall satisfaction were examined.

\subsection{Analysis}

The collected data were analyzed using IBM SPSS 23 statistical software. A cluster analysis was performed to divide the participants into three groups (high, medium, low) according to height and BMI. In the case of BMI groups, the WHO Asia-Pacific guideline of BMI was used to classify the degree of obesity [40], with "underweight" being less than $18.5 \mathrm{~kg} \cdot \mathrm{m}^{-2}$, "normal weight" being $18.5-22.9 \mathrm{~kg} \cdot \mathrm{m}^{-2}$, "overweight" being 23-24.9 $\mathrm{kg} \cdot \mathrm{m}^{-2}$ and "obese" being more than $25 \mathrm{~kg} \cdot \mathrm{m}^{-2}$. One-way ANOVA was performed to analyze differences of sleep habits, sleeping symptoms and the comfort and support characteristics of pillows according to age, height, BMI and pillow types. As a post-hoc analysis, Fisher's least significant difference (LSD) test was used to identify differences between pillow-use groups. Independent $t$-tests were performed to analyze differences according to sex and sleeping position. In addition, the scores of each variable were compared by statistical analysis based on means and standard deviation. To explain the relationship between symptoms during sleep and after waking and quality of sleep, a multiple linear regression analysis was performed. The $p$-values less than 0.05 were considered statistically significant. 


\section{Results}

Three hundred and thirty-two adults aged 20-76 years responded to our study survey. The mean age of participants was 41.6 years (male: Mean $=40.4$ years, $S D=15.2$ years; female: Mean $=42.9$ years, $\mathrm{SD}=15.4$ years). Participants were classified into three age groups: $178(54 \%)$ people in their 20s-30s, 81 (24\%) in their 40s-50s and 73 (22\%) in their 60s-70s. There were $176(53 \%)$ male and 156 $(47 \%)$ female participants. Height was classified into three groups: $107(33 \%)$ people with a short stature (less than $162.5 \mathrm{~cm}), 134(40 \%)$ with a medium stature $(162.5-174 \mathrm{~cm})$ and $91(26 \%)$ with a tall stature (more than $174 \mathrm{~cm}$ ). Body Mass Index (BMI) was also classified into three groups: $142(43 \%)$ people with a low BMI (less than $\left.22.4 \mathrm{~kg} \cdot \mathrm{m}^{-2}\right), 143(43 \%)$ with a medium BMI $\left(22.4-26.7 \mathrm{~kg} \cdot \mathrm{m}^{-2}\right)$ and $47(14 \%)$ with a high BMI (more than $\left.26.7 \mathrm{~kg} \cdot \mathrm{m}^{-2}\right)$.

\subsection{Sleep Habits}

Descriptive statistics regarding the sleep habits and sleeping symptoms of participants are presented in Table 3. There were significant differences in the number of tossing and turning or waking events, wake-up times and sleep durations among age groups $(p<0.05$; Table 3$)$. The number of tossing and turning or waking events during sleep increased with age.

Table 3. T-test and ANOVA results for sleep habits (Mean (SD)).

\begin{tabular}{|c|c|c|c|c|c|c|c|c|c|c|c|c|c|}
\hline & \multirow{2}{*}{ Sleep Habits } & \multirow{2}{*}{ Total } & \multicolumn{3}{|c|}{ Age (Years) } & \multicolumn{2}{|c|}{ Sex } & \multicolumn{3}{|c|}{ Height } & \multicolumn{3}{|c|}{ BMI } \\
\hline & & & $20-39$ & $40-59$ & $60-76$ & $\mathbf{M}$ & $\mathbf{F}$ & S & $\mathbf{M}$ & $\mathrm{T}$ & $\mathbf{L}$ & $\mathbf{M}$ & $\mathbf{H}$ \\
\hline \multirow{5}{*}{ Sleep habits } & $\begin{array}{l}\text { Toss and turn } \\
\text { or waking up } \\
\text { (number) }\end{array}$ & $\begin{array}{c}2.0 \\
(1.1)\end{array}$ & $\begin{array}{l}1.6^{*} \\
(0.9)\end{array}$ & $\begin{array}{l}2.3^{*} \\
(0.7)\end{array}$ & $\begin{array}{l}2.7^{*} \\
(1.1)\end{array}$ & $\begin{array}{c}2.0 \\
(1.1)\end{array}$ & $\begin{array}{c}2.1 \\
(2.1)\end{array}$ & $\begin{array}{l}2.3^{*} \\
(1.1)\end{array}$ & $\begin{array}{l}2.0^{*} \\
(1.1)\end{array}$ & $\begin{array}{l}1.7^{*} \\
(0.9)\end{array}$ & $\begin{array}{l}1.9^{*} \\
(1.0)\end{array}$ & $\begin{array}{l}2.2 * \\
(1.1)\end{array}$ & $\begin{array}{l}2.0^{*} \\
(1.0)\end{array}$ \\
\hline & $\begin{array}{l}\text { Sleep latency } \\
\text { (minutes) }\end{array}$ & $\begin{array}{l}28.6 \\
(25.0)\end{array}$ & $\begin{array}{c}30.1 \\
(21.5)\end{array}$ & $\begin{array}{c}25.9 \\
(14.2)\end{array}$ & $\begin{array}{c}28.1 \\
(29.3)\end{array}$ & $\begin{array}{c}27.4 \\
(19.1)\end{array}$ & $\begin{array}{l}29.9 \\
(25)\end{array}$ & $\begin{array}{l}30.5 \\
(26.6)\end{array}$ & $\begin{array}{c}27.1 \\
(18.7)\end{array}$ & $\begin{array}{l}28.6 \\
(20.7)\end{array}$ & $\begin{array}{l}30.6 \\
(26.8)\end{array}$ & $\begin{array}{c}28.0 \\
(18.7)\end{array}$ & $\begin{array}{c}24.4 \\
(14.1)\end{array}$ \\
\hline & Bedtime (time) & $\begin{array}{l}24.1 \\
(1.4)\end{array}$ & $\begin{array}{c}24.8 \\
* \\
(1.5)\end{array}$ & $\begin{array}{c}23.6 \\
* \\
(1.1)\end{array}$ & $\begin{array}{c}22.9 \\
*(1.1)\end{array}$ & $\begin{array}{c}24.2 \\
* \\
(1.7) \\
\end{array}$ & $\begin{array}{c}23.9 \\
* \\
(1.4) \\
\end{array}$ & $\begin{array}{c}23.7 \\
* \\
(1.4) \\
\end{array}$ & $\begin{array}{c}24.0 \\
* \\
(1.5)\end{array}$ & $\begin{array}{c}24.8 \\
* \\
(1.6) \\
\end{array}$ & $\begin{array}{l}24.2 \\
(1.6)\end{array}$ & $\begin{array}{l}24.0 \\
(1.6)\end{array}$ & $\begin{array}{l}24.2 \\
(1.5)\end{array}$ \\
\hline & $\begin{array}{l}\text { Wake-up time } \\
\quad\left(\text { time }^{\text {a }}\right)\end{array}$ & $\begin{array}{c}7.0 \\
(1.6) \\
\end{array}$ & $\begin{array}{l}7.9^{*} \\
(1.4) \\
\end{array}$ & $\begin{array}{l}6.2^{*} \\
(0.9) \\
\end{array}$ & $\begin{array}{l}5.8^{*} \\
(2.1) \\
\end{array}$ & $\begin{array}{c}7.1 \\
(1.9) \\
\end{array}$ & $\begin{array}{c}6.9 \\
(1.6) \\
\end{array}$ & $\begin{array}{l}6.5^{*} \\
(1.6) \\
\end{array}$ & $\begin{array}{l}7.0^{*} \\
(2.0) \\
\end{array}$ & $\begin{array}{l}7.6^{*} \\
(1.5) \\
\end{array}$ & $\begin{array}{c}7.2 \\
(1.7) \\
\end{array}$ & $\begin{array}{c}6.9 \\
(2.0) \\
\end{array}$ & $\begin{array}{c}6.8 \\
(1.5) \\
\end{array}$ \\
\hline & $\begin{array}{l}\text { Sleep duration } \\
\text { (hours) }\end{array}$ & $\begin{array}{c}6.8 \\
(1.1) \\
\end{array}$ & $\begin{array}{l}7.0^{*} \\
(1.2) \\
\end{array}$ & $\begin{array}{l}6.6^{*} \\
(1.0) \\
\end{array}$ & $\begin{array}{l}6.7^{*} \\
(1.1) \\
\end{array}$ & $\begin{array}{c}6.7 \\
(1.2) \\
\end{array}$ & $\begin{array}{c}6.9 \\
(1.1) \\
\end{array}$ & $\begin{array}{c}6.8 \\
(1.0) \\
\end{array}$ & $\begin{array}{c}6.8 \\
(1.2) \\
\end{array}$ & $\begin{array}{c}6.8 \\
(1.2) \\
\end{array}$ & $\begin{array}{l}7.0^{*} \\
(1.2) \\
\end{array}$ & $\begin{array}{l}6.7^{*} \\
(1.1) \\
\end{array}$ & $\begin{array}{l}6.6^{*} \\
(1.0) \\
\end{array}$ \\
\hline \multirow{3}{*}{$\begin{array}{l}\text { Symptoms } \\
\text { during sleep }\end{array}$} & $\begin{array}{l}\text { Comfort Position } \\
\text { changes }\end{array}$ & $\begin{array}{c}4.9 \\
(1.5)\end{array}$ & $\begin{array}{c}4.9 \\
(1.5)\end{array}$ & $\begin{array}{c}4.8 \\
(1.3)\end{array}$ & $\begin{array}{c}4.7 \\
(1.3)\end{array}$ & $\begin{array}{c}4.9 \\
(1.4)\end{array}$ & $\begin{array}{c}4.8 \\
(1.5)\end{array}$ & $\begin{array}{c}4.8 \\
(1.4)\end{array}$ & $\begin{array}{c}4.8 \\
(1.5)\end{array}$ & $\begin{array}{c}5.1 \\
(1.3)\end{array}$ & $\begin{array}{c}4.8 \\
(1.5)\end{array}$ & $\begin{array}{c}5.0 \\
(1.3)\end{array}$ & $\begin{array}{c}4.7 \\
(1.3) \\
\end{array}$ \\
\hline & Less Snoring or coughing & $\begin{array}{c}4.8 \\
(1.5) \\
\end{array}$ & $\begin{array}{l}5.1^{*} \\
(1.6) \\
\end{array}$ & $\begin{array}{l}4.5^{*} \\
(1.6) \\
\end{array}$ & $\begin{array}{l}46^{*} \\
(1.5) \\
\end{array}$ & $\begin{array}{c}4.6 \\
(1.6) \\
\end{array}$ & $\begin{array}{c}5.1 \\
(1.5) \\
\end{array}$ & $\begin{array}{c}4.9 \\
(1.6) \\
\end{array}$ & $\begin{array}{c}4.8 \\
(1.6) \\
\end{array}$ & $\begin{array}{c}4.8 \\
(1.6) \\
\end{array}$ & $\begin{array}{l}5.2 * \\
(1.6) \\
\end{array}$ & $\begin{array}{l}4.8^{*} \\
(1.5) \\
\end{array}$ & $\begin{array}{l}4.1^{*} \\
(1.6) \\
\end{array}$ \\
\hline & Breathing & $\begin{array}{c}5.3 \\
(1.4) \\
\end{array}$ & $\begin{array}{l}5.6^{*} \\
(1.3) \\
\end{array}$ & $\begin{array}{l}5.1^{*} \\
(1.4)\end{array}$ & $\begin{array}{l}4.9^{*} \\
(1.5)\end{array}$ & $\begin{array}{c}5.2 \\
(1.4) \\
\end{array}$ & $\begin{array}{c}5.4 \\
(1.4) \\
\end{array}$ & $\begin{array}{c}5.4 \\
(1.3) \\
\end{array}$ & $\begin{array}{c}5.2 \\
(1.5) \\
\end{array}$ & $\begin{array}{c}5.4 \\
(1.4)\end{array}$ & $\begin{array}{l}5.4^{*} \\
(1.4)\end{array}$ & $\begin{array}{l}5.3^{*} \\
(1.4) \\
\end{array}$ & $\begin{array}{l}4.8^{*} \\
(1.4)\end{array}$ \\
\hline \multirow{4}{*}{$\begin{array}{l}\text { Symptoms } \\
\text { after waking }\end{array}$} & Less Head pain & $\begin{array}{c}5.4 \\
(1.3)\end{array}$ & $\begin{array}{l}5.5^{*} \\
(1.3)\end{array}$ & $\begin{array}{l}5.3^{*} \\
(1.2)\end{array}$ & $\begin{array}{l}5.1 * \\
(1.3)\end{array}$ & $\begin{array}{c}5.5 \\
(1.3)\end{array}$ & $\begin{array}{c}5.3 \\
(1.2)\end{array}$ & $\begin{array}{l}5.2 * \\
(1.3)\end{array}$ & $\begin{array}{l}5.4^{*} \\
(1.3)\end{array}$ & $\begin{array}{l}5.6^{*} \\
(1.2)\end{array}$ & $\begin{array}{c}5.4 \\
(1.2)\end{array}$ & $\begin{array}{c}5.3 \\
(1.3)\end{array}$ & $\begin{array}{c}5.4 \\
(1.3)\end{array}$ \\
\hline & Less Neck pain & $\begin{array}{c}4.9 \\
(1.4)\end{array}$ & $\begin{array}{c}4.8 \\
(1.6)\end{array}$ & $\begin{array}{c}5.0 \\
(1.2)\end{array}$ & $\begin{array}{l}5.0 \\
(1.4)\end{array}$ & $\begin{array}{l}5.0 \\
(1.5)\end{array}$ & $\begin{array}{c}4.7 \\
(1.4)\end{array}$ & $\begin{array}{c}4.7 \\
(1.4)\end{array}$ & $\begin{array}{l}5.0 \\
(1.4)\end{array}$ & $\begin{array}{l}5.0 \\
(1.6)\end{array}$ & $\begin{array}{c}4.9 \\
(1.4)\end{array}$ & $\begin{array}{c}4.8 \\
(1.4)\end{array}$ & $\begin{array}{c}5.0 \\
(1.4)\end{array}$ \\
\hline & Less Shoulder pain & $\begin{array}{c}4.7 \\
(1.5) \\
\end{array}$ & $\begin{array}{c}4.7 \\
(1.6) \\
\end{array}$ & $\begin{array}{c}4.7 \\
(1.2) \\
\end{array}$ & $\begin{array}{c}4.7 \\
(1.4) \\
\end{array}$ & $\begin{array}{c}4.8 \\
(1.4) \\
\end{array}$ & $\begin{array}{c}4.5 \\
(1.5) \\
\end{array}$ & $\begin{array}{c}4.6 \\
(1.5) \\
\end{array}$ & $\begin{array}{c}4.6 \\
(1.5) \\
\end{array}$ & $\begin{array}{c}4.9 \\
(1.4) \\
\end{array}$ & $\begin{array}{c}4.7 \\
(1.5) \\
\end{array}$ & $\begin{array}{c}4.6 \\
(1.5) \\
\end{array}$ & $\begin{array}{c}4.9 \\
(1.4) \\
\end{array}$ \\
\hline & $\begin{array}{l}\text { Less Sleepiness during } \\
\text { waking hours }\end{array}$ & $\begin{array}{c}4.4 \\
(1.4)\end{array}$ & $\begin{array}{c}4.4 \\
(1.5)\end{array}$ & $\begin{array}{c}4.4 \\
(1.4)\end{array}$ & $\begin{array}{c}4.5 \\
(1.3)\end{array}$ & $\begin{array}{c}4.4 \\
(1.4)\end{array}$ & $\begin{array}{c}4.5 \\
(1.4)\end{array}$ & $\begin{array}{c}4.5 \\
(1.3)\end{array}$ & $\begin{array}{c}4.3 \\
(1.4)\end{array}$ & $\begin{array}{c}4.6 \\
(1.5)\end{array}$ & $\begin{array}{c}4.6 \\
(1.5)\end{array}$ & $\begin{array}{c}4.3 \\
(1.4)\end{array}$ & $\begin{array}{c}4.5 \\
(1.4)\end{array}$ \\
\hline \multirow{4}{*}{ Less Fatigue } & Head & $\begin{array}{c}5.1 \\
(1.2) \\
\end{array}$ & $\begin{array}{c}5.2 \\
(1.3) \\
\end{array}$ & $\begin{array}{c}5.0 \\
(1.1) \\
\end{array}$ & $\begin{array}{c}5.0 \\
(1.2) \\
\end{array}$ & $\begin{array}{c}5.1 \\
(1.2) \\
\end{array}$ & $\begin{array}{c}5.1 \\
(1.2) \\
\end{array}$ & $\begin{array}{c}5.0 \\
(1.2) \\
\end{array}$ & $\begin{array}{c}5.1 \\
(1.2) \\
\end{array}$ & $\begin{array}{c}5.2 \\
(1.1) \\
\end{array}$ & $\begin{array}{c}5.0 \\
(1.3) \\
\end{array}$ & $\begin{array}{c}5.2 \\
(1.1) \\
\end{array}$ & $\begin{array}{c}5.0 \\
(1.0) \\
\end{array}$ \\
\hline & Neck & $\begin{array}{c}4.6 \\
(1.4)\end{array}$ & $\begin{array}{l}4.4^{*} \\
(1.5)\end{array}$ & $\begin{array}{l}4.9^{*} \\
(1.2)\end{array}$ & $\begin{array}{l}4.9^{*} \\
(1.2)\end{array}$ & $\begin{array}{c}4.7 \\
(1.4)\end{array}$ & $\begin{array}{c}4.5 \\
(1.4)\end{array}$ & $\begin{array}{c}4.6 \\
(1.4)\end{array}$ & $\begin{array}{c}4.6 \\
(1.4)\end{array}$ & $\begin{array}{c}4.6 \\
(1.5)\end{array}$ & $\begin{array}{c}4.5 \\
(1.4)\end{array}$ & $\begin{array}{c}4.7 \\
(1.5)\end{array}$ & $\begin{array}{c}4.6 \\
(1.3)\end{array}$ \\
\hline & Shoulder & $\begin{array}{c}4.2 \\
(1.4) \\
\end{array}$ & $\begin{array}{l}4.1^{*} \\
(1.5) \\
\end{array}$ & $\begin{array}{l}4.4^{*} \\
(1.3) \\
\end{array}$ & $\begin{array}{l}4.5^{*} \\
(1.4) \\
\end{array}$ & $\begin{array}{c}4.3 \\
(1.5) \\
\end{array}$ & $\begin{array}{c}4.2 \\
(1.4) \\
\end{array}$ & $\begin{array}{c}4.3 \\
(1.4) \\
\end{array}$ & $\begin{array}{c}4.2 \\
(1.5) \\
\end{array}$ & $\begin{array}{c}4.2 \\
(1.4) \\
\end{array}$ & $\begin{array}{c}4.3 \\
(1.4) \\
\end{array}$ & $\begin{array}{c}4.3 \\
(1.5) \\
\end{array}$ & $\begin{array}{c}4.2 \\
(1.3) \\
\end{array}$ \\
\hline & Sleep quality & $\begin{array}{c}4.8 \\
(1.3)\end{array}$ & $\begin{array}{c}4.9 \\
(1.3)\end{array}$ & $\begin{array}{c}4.8 \\
(1.3)\end{array}$ & $\begin{array}{c}4.7 \\
(1.2)\end{array}$ & $\begin{array}{c}4.9 \\
(1.3)\end{array}$ & $\begin{array}{c}4.8 \\
(1.3)\end{array}$ & $\begin{array}{l}4.7^{*} \\
(1.2)\end{array}$ & $\begin{array}{l}4.8^{*} \\
(1.3)\end{array}$ & $\begin{array}{l}5.1^{*} \\
(1.2)\end{array}$ & $\begin{array}{c}4.9 \\
(1.3)\end{array}$ & $\begin{array}{c}4.9 \\
(1.3)\end{array}$ & $\begin{array}{c}4.6 \\
(1.2)\end{array}$ \\
\hline
\end{tabular}

* Analysis of variance (age, height, BMI) and independent $t$-test value (sex), $p \leq 0.05$; ${ }^{\text {T }}$ Time is expressed using a 24-h clock.

There were significant differences in bedtime according to sex $(p<0.05$; Table 3). Females went to bed earlier than males. Older individuals went to sleep earlier and woke up earlier. Sleep duration was shortest for participants in their 40s-50s. Subjective sleep evaluation indicated that the older an individual was, the more snoring or coughing symptoms and greater discomfort with breathing they 
experienced during sleep. Many participants reported feeling sleepy during waking hours. Head pain after waking was not a common complaint, but older participants experienced more head pain than younger individuals.

Sleep habits differed significantly according to height and BMI groups ( $p<0.05$; Table 3$)$. The tallest group had the lowest number of tossing and turning or waking events and the earliest bedtime and wake-up time. The tallest participants felt the least head pain after sleeping (high score). The overall sleep quality was also the highest for the tallest individuals. There were no significant differences in sleeping position according to height. The medium BMI class showed the highest number of tossing and turning or waking events, and the highest BMI group had the shortest sleep duration. In addition, individuals in the high BMI class had poorer scores for snoring or coughing symptoms and discomfort with breathing during sleep than medium and low BMI classes. There were no significant differences in sleeping position by BMI.

The most common sleeping position was "Mainly sleep in a supine position but also sleep in a lateral position" (44\%), with 9\% of participants reporting sleeping mainly in a supine position (Table 4). Movement from a supine to a lateral position or vice versa was more common than a fixed sleeping position. Overall, a supine position was more common than a lateral position. Although most males and females slept mainly in a supine position, females slept more often on their backs or sides than males, and males slept more often in a supine position.

Table 4. Descriptive statistics of sleeping position.

\begin{tabular}{|c|c|c|c|c|c|}
\hline Position & $n$ & Detailed Descriptions of Position & $n$ & Male & Female \\
\hline \multirow{3}{*}{ Supine } & \multirow{2}{*}{$174(52 \%)$} & Mainly sleep in a supine position & $29(9 \%)$ & $21(72 \%)$ & $8(28 \%)$ \\
\hline & & $\begin{array}{l}\text { Mainly sleep in a supine position but also } \\
\text { sleep in a lateral position }\end{array}$ & $145(44 \%)$ & $84(58 \%)$ & $61(42 \%)$ \\
\hline & & & & $105(60 \%)$ & $69(47 \%)$ \\
\hline \multirow{3}{*}{ Lateral } & \multirow{2}{*}{$146(44 \%)$} & Mainly sleep in a lateral position & $58(18 \%)$ & $31(53 \%)$ & $27(47 \%)$ \\
\hline & & $\begin{array}{l}\text { Mainly sleep in a lateral position but also } \\
\text { sleep in a supine position }\end{array}$ & $88(27 \%)$ & $38(43 \%)$ & $50(57 \%)$ \\
\hline & & & & $69(40 \%)$ & $77(53 \%)$ \\
\hline \multirow[t]{2}{*}{ Other } & $12(4 \%)$ & Prone position, etc. & $12(4 \%)$ & $2(17 \%)$ & $10(83 \%)$ \\
\hline & & Total & 332 & 176 & 156 \\
\hline
\end{tabular}

\subsection{Comfort and Support Characteristics of Pillows}

Most participants used regular-type pillows (54\%), but 22\% of participants used contour-type pillows (peanut-shaped pillow: 8\%; functional A-type pillow: 7\%; functional B-type pillow: $8 \%$; other: $2 \%$ ). The score for the pillow in use was lowest for shoulder support, followed by shoulder fatigue (Table 5). The support and comfort questions scored higher on the head than the shoulders and neck. There were significant differences in shoulder support, neck fatigue and pillow temperature satisfaction among the age groups $(p<0.05$; Table 5). Participants in their 20s-30s reported lower scores for shoulder support (tended to feel the least support) and neck fatigue (tended to feel the least fatigue) than the other two age groups. A low satisfaction score was reported for the older group. Neck support and shape suitability of the pillow for the neck and shoulder differed significantly by sex. Females had lower scores than males for these questions, indicating less satisfaction with neck support, shape suitability and shoulder support of their pillows. There were also significant differences in head support, head fatigue and shape suitability of the head and neck support according to sleeping position ( $p<0.05$; Table 5). Participants who slept in a lateral position had lower scores for these items (i.e., received less support and experienced more fatigue and less shape suitability) than those who slept in a supine position. 
Table 5. T-test and ANOVA results for comfort and support characteristics of pillows (Mean (S.D.)).

\begin{tabular}{|c|c|c|c|c|c|c|c|c|c|}
\hline \multirow{2}{*}{ Variables } & & \multirow{2}{*}{ Total } & \multicolumn{3}{|c|}{ Age (Years) } & \multicolumn{2}{|c|}{ Sex } & \multicolumn{2}{|c|}{ Sleeping Position } \\
\hline & & & $20-39$ & $40-59$ & $60-76$ & Male & Female & Supine & Lateral \\
\hline \multirow{3}{*}{ Support } & Head & $\begin{array}{c}5.1 \\
(1.2)\end{array}$ & $\begin{array}{c}5.2 \\
(1.3)\end{array}$ & $\begin{array}{c}5.2 \\
(1.1)\end{array}$ & $\begin{array}{c}5.0 \\
(1.2)\end{array}$ & $\begin{array}{c}5.1 \\
(1.3)\end{array}$ & $\begin{array}{c}5.1 \\
(1.2)\end{array}$ & $\begin{array}{l}5.3^{*} \\
(1.1)\end{array}$ & $\begin{array}{l}4.9^{*} \\
(1.3)\end{array}$ \\
\hline & Neck & $\begin{array}{c}4.7 \\
(1.4) \\
\end{array}$ & $\begin{array}{c}4.6 \\
(1.5) \\
\end{array}$ & $\begin{array}{c}4.9 \\
(1.2) \\
\end{array}$ & $\begin{array}{c}4.9 \\
(1.3) \\
\end{array}$ & $\begin{array}{l}4.6^{*} \\
(1.5) \\
\end{array}$ & $\begin{array}{l}4.9^{*} \\
(1.3)\end{array}$ & $\begin{array}{l}4.9^{*} \\
(1.4)\end{array}$ & $\begin{array}{l}4.5^{*} \\
(1.4)\end{array}$ \\
\hline & Shoulder & $\begin{array}{c}3.8 \\
(1.5)\end{array}$ & $\begin{array}{l}3.6^{*} \\
(1.5)\end{array}$ & $\begin{array}{l}4.1^{*} \\
(1.4)\end{array}$ & $\begin{array}{l}4.1^{*} \\
(1.4)\end{array}$ & $\begin{array}{c}3.7 \\
(1.5)\end{array}$ & $\begin{array}{c}3.9 \\
(1.5) \\
\end{array}$ & $\begin{array}{c}3.9 \\
(1.5)\end{array}$ & $\begin{array}{c}3.7 \\
(1.5) \\
\end{array}$ \\
\hline \multirow{3}{*}{ Comfort } & Head & $\begin{array}{c}5.2 \\
(1.2)\end{array}$ & $\begin{array}{c}5.2 \\
(1.3)\end{array}$ & $\begin{array}{c}5.3 \\
(1.1)\end{array}$ & $\begin{array}{c}5.0 \\
(1.2)\end{array}$ & $\begin{array}{c}5.2 \\
(1.2)\end{array}$ & $\begin{array}{c}5.2 \\
(1.3)\end{array}$ & $\begin{array}{c}5.2 \\
(1.3)\end{array}$ & $\begin{array}{c}5.2 \\
(1.2)\end{array}$ \\
\hline & Neck & $\begin{array}{c}4.7 \\
(1.4)\end{array}$ & $\begin{array}{c}4.6 \\
(1.5)\end{array}$ & $\begin{array}{c}5.0 \\
(1.2)\end{array}$ & $\begin{array}{c}4.8 \\
(1.4)\end{array}$ & $\begin{array}{c}4.7 \\
(1.5)\end{array}$ & $\begin{array}{c}4.7 \\
(1.3)\end{array}$ & $\begin{array}{c}4.9 \\
(1.4)\end{array}$ & $\begin{array}{c}4.6 \\
(1.3)\end{array}$ \\
\hline & Shoulder & $\begin{array}{c}4.1 \\
(1.5)\end{array}$ & $\begin{array}{c}3.9 \\
(1.5)\end{array}$ & $\begin{array}{c}4.3 \\
(1.4) \\
\end{array}$ & $\begin{array}{c}4.1 \\
(1.4)\end{array}$ & $\begin{array}{c}4.0 \\
(1.5)\end{array}$ & $\begin{array}{c}4.2 \\
(1.4)\end{array}$ & $\begin{array}{c}4.2 \\
(1.5)\end{array}$ & $\begin{array}{c}3.9 \\
(1.4)\end{array}$ \\
\hline \multirow{3}{*}{ Less Fatigue } & Head & $\begin{array}{c}5.1 \\
(1.2)\end{array}$ & $\begin{array}{c}5.2 \\
(1.3)\end{array}$ & $\begin{array}{c}5.0 \\
(1.1)\end{array}$ & $\begin{array}{c}5.0 \\
(1.2)\end{array}$ & $\begin{array}{c}5.1 \\
(1.2)\end{array}$ & $\begin{array}{c}5.1 \\
(1.2)\end{array}$ & $\begin{array}{c}5.2 \\
(1.2)\end{array}$ & $\begin{array}{c}5 \\
(1.2) \\
\end{array}$ \\
\hline & Neck & $\begin{array}{c}4.6 \\
(1.4)\end{array}$ & $\begin{array}{l}4.4^{*} \\
(1.5)\end{array}$ & $\begin{array}{l}4.9^{*} \\
(1.2)\end{array}$ & $\begin{array}{l}4.9^{*} \\
(1.2)\end{array}$ & $\begin{array}{c}4.7 \\
(1.4)\end{array}$ & $\begin{array}{c}4.5 \\
(1.4)\end{array}$ & $\begin{array}{l}4.8^{*} \\
(1.4)\end{array}$ & $\begin{array}{l}4.4^{*} \\
(1.5)\end{array}$ \\
\hline & Shoulder & $\begin{array}{c}4.2 \\
(1.4)\end{array}$ & $\begin{array}{l}4.1^{*} \\
(1.5)\end{array}$ & $\begin{array}{l}4.4^{*} \\
(1.3)\end{array}$ & $\begin{array}{l}4.5^{*} \\
(1.4)\end{array}$ & $\begin{array}{c}4.3 \\
(1.5)\end{array}$ & $\begin{array}{c}4.2 \\
(1.4)\end{array}$ & $\begin{array}{c}4.4 \\
(1.5)\end{array}$ & $\begin{array}{c}4.1 \\
(1.4)\end{array}$ \\
\hline \multirow{2}{*}{ Height suitability } & Head & $\begin{array}{c}5.0 \\
(1.3)\end{array}$ & $\begin{array}{c}5.0 \\
(1.4)\end{array}$ & $\begin{array}{c}5.0 \\
(1.3)\end{array}$ & $\begin{array}{c}5.1 \\
(1.3)\end{array}$ & $\begin{array}{c}5.0 \\
(1.3)\end{array}$ & $\begin{array}{c}5.0 \\
(1.3)\end{array}$ & $\begin{array}{c}5.1 \\
(1.3)\end{array}$ & $\begin{array}{c}4.8 \\
(1.3)\end{array}$ \\
\hline & Neck & $\begin{array}{c}4.7 \\
(1.4)\end{array}$ & $\begin{array}{c}4.6 \\
(1.4)\end{array}$ & $\begin{array}{c}4.7 \\
(1.4)\end{array}$ & $\begin{array}{c}4.9 \\
(1.3)\end{array}$ & $\begin{array}{c}4.7 \\
(1.5)\end{array}$ & $\begin{array}{c}4.7 \\
(1.3)\end{array}$ & $\begin{array}{c}4.8 \\
(1.4)\end{array}$ & $\begin{array}{c}4.5 \\
(1.4)\end{array}$ \\
\hline \multirow{3}{*}{ Shape suitability } & Head & $\begin{array}{c}5.1 \\
(1.3)\end{array}$ & $\begin{array}{c}5.1 \\
(1.3)\end{array}$ & $\begin{array}{c}5.1 \\
(1.2)\end{array}$ & $\begin{array}{c}5.0 \\
(1.2)\end{array}$ & $\begin{array}{c}5.1 \\
(1.2)\end{array}$ & $\begin{array}{c}5.0 \\
(1.3)\end{array}$ & $\begin{array}{l}5.2^{*} \\
(1.2)\end{array}$ & $\begin{array}{l}4.9^{*} \\
(1.3)\end{array}$ \\
\hline & Neck & $\begin{array}{c}4.8 \\
(1.4) \\
\end{array}$ & $\begin{array}{c}4.7 \\
(1.4) \\
\end{array}$ & $\begin{array}{c}4.9 \\
(1.3) \\
\end{array}$ & $\begin{array}{c}4.9 \\
(1.3)\end{array}$ & $\begin{array}{l}4.7^{*} \\
(1.4)\end{array}$ & $\begin{array}{l}4.8^{*} \\
(1.2)\end{array}$ & $\begin{array}{c}4.8 \\
(1.3)\end{array}$ & $\begin{array}{c}4.7 \\
(1.4)\end{array}$ \\
\hline & Shoulder & $\begin{array}{c}4.4 \\
(1.4)\end{array}$ & $\begin{array}{c}4.4 \\
(1.5)\end{array}$ & $\begin{array}{c}4.5 \\
(1.4)\end{array}$ & $\begin{array}{c}4.4 \\
(1.2) \\
\end{array}$ & $\begin{array}{l}4.3^{*} \\
(1.5)\end{array}$ & $\begin{array}{l}4.4^{*} \\
(1.3)\end{array}$ & $\begin{array}{c}4.5 \\
(1.3)\end{array}$ & $\begin{array}{c}4.3 \\
(1.5)\end{array}$ \\
\hline \multicolumn{2}{|c|}{ Temperature suitability } & $\begin{array}{c}5.0 \\
(1.3)\end{array}$ & $\begin{array}{l}5.2 * \\
(1.2)\end{array}$ & $\begin{array}{l}4.8^{*} \\
(1.3)\end{array}$ & $\begin{array}{l}4.7^{*} \\
(1.4)\end{array}$ & $\begin{array}{c}5.0 \\
(1.3)\end{array}$ & $\begin{array}{c}5.0 \\
(1.3)\end{array}$ & $\begin{array}{c}5.1 \\
(1.4)\end{array}$ & $\begin{array}{c}4.9 \\
(1.4)\end{array}$ \\
\hline \multicolumn{2}{|c|}{ Overall satisfaction } & $\begin{array}{c}4.9 \\
(1.3)\end{array}$ & $\begin{array}{c}4.9 \\
(1.4)\end{array}$ & $\begin{array}{c}4.8 \\
(1.2)\end{array}$ & $\begin{array}{c}4.9 \\
(1.2)\end{array}$ & $\begin{array}{c}4.9 \\
(1.3)\end{array}$ & $\begin{array}{c}4.8 \\
(1.3)\end{array}$ & $\begin{array}{c}5.0 \\
(1.2)\end{array}$ & $\begin{array}{c}4.8 \\
(1.4)\end{array}$ \\
\hline \multicolumn{2}{|c|}{ Willingness to repurchase } & $\begin{array}{c}4.6 \\
(1.6)\end{array}$ & $\begin{array}{c}4.4 \\
(1.6)\end{array}$ & $\begin{array}{c}4.8 \\
(1.5)\end{array}$ & $\begin{array}{c}4.7 \\
(1.6)\end{array}$ & $\begin{array}{c}4.5 \\
(1.6)\end{array}$ & $\begin{array}{c}4.6 \\
(1.6)\end{array}$ & $\begin{array}{c}4.7 \\
(1.6)\end{array}$ & $\begin{array}{c}4.4 \\
(1.6)\end{array}$ \\
\hline
\end{tabular}

* Analysis of variance (age), independent $t$-test value (sex, sleeping position), $p \leq 0.05$.

Support, comfort and shape suitability of neck and shoulder, head support and height suitability of neck differed significantly by pillow types $(p<0.05)$ according to the ANOVA results of comfort and support factors by pillow types (Table 6). Participants who used functional-type pillows reported greater head, neck and shoulder support and neck comfort than those who used other pillow types. Participants who used a peanut-shaped pillow had the highest shoulder comfort. Participants who used contour-type pillows and functional A-type pillows had the highest scores for neck height suitability. The functional A-type pillows received the highest shape-suitability score for the neck, whereas the peanut-shaped pillows received the highest shape-suitability score for the shoulders. Regular-type pillows scored the lowest on several questions. 
Table 6. ANOVA results of comfort and support by pillow types (Mean (SD)).

\begin{tabular}{|c|c|c|c|c|c|c|c|}
\hline \multicolumn{2}{|c|}{ Variables } & \multirow{2}{*}{$\begin{array}{c}\text { Regular Pillow } \\
4.9^{*} \\
(1.3) \\
\end{array}$} & \multirow{2}{*}{$\begin{array}{c}\text { Contour Pillow } \\
5.3^{*} \\
(1.1) \\
\end{array}$} & \multirow{2}{*}{$\begin{array}{c}\text { Peanut-Shaped Pillow } \\
5.2^{*} \\
(1.2)\end{array}$} & \multirow{2}{*}{$\begin{array}{c}\text { Functional A Pillow } \\
5.6^{*} \\
(1.0) \\
\end{array}$} & \multirow{2}{*}{$\begin{array}{c}\text { Functional B Pillow } \\
5.5^{*} \\
(1.2) \\
\end{array}$} & \multirow{2}{*}{$\begin{array}{c}\text { Other } \\
6.0^{*} \\
(0.6)\end{array}$} \\
\hline & Head & & & & & & \\
\hline \multirow[t]{2}{*}{ Support } & Neck & $\begin{array}{l}4.3^{*} \\
(1.5) \\
\end{array}$ & $\begin{array}{l}5.1^{*} \\
(1.1) \\
\end{array}$ & $\begin{array}{l}5.2^{*} \\
(1.0) \\
\end{array}$ & $\begin{array}{l}5.5^{*} \\
(1.1) \\
\end{array}$ & $\begin{array}{l}5.5^{*} \\
(0.9) \\
\end{array}$ & $\begin{array}{l}5.3^{*} \\
(1.1) \\
\end{array}$ \\
\hline & Shoulder & $\begin{array}{l}3.5^{*} \\
(1.5) \\
\end{array}$ & $\begin{array}{l}4.2 * \\
(1.3) \\
\end{array}$ & $\begin{array}{l}4.1^{*} \\
(1.6) \\
\end{array}$ & $\begin{array}{l}4.1^{*} \\
(1.3) \\
\end{array}$ & $\begin{array}{l}4.7^{*} \\
(1.4) \\
\end{array}$ & $\begin{array}{l}3.6^{*} \\
(1.6) \\
\end{array}$ \\
\hline \multirow{3}{*}{ Comfort } & Head & $\begin{array}{c}5.1 \\
(1.3)\end{array}$ & $\begin{array}{c}5.3 \\
(1.1)\end{array}$ & $\begin{array}{c}5.4 \\
(1.1)\end{array}$ & $\begin{array}{c}5.5 \\
(0.9)\end{array}$ & $\begin{array}{c}5.2 \\
(1.4)\end{array}$ & $\begin{array}{c}4.9 \\
(1.1)\end{array}$ \\
\hline & Neck & $\begin{array}{l}4.4^{*} \\
(1.5) \\
\end{array}$ & $\begin{array}{l}5.0^{*} \\
(1.1) \\
\end{array}$ & $\begin{array}{l}5.0^{*} \\
(1.3) \\
\end{array}$ & $\begin{array}{l}5.3^{*} \\
(1.1) \\
\end{array}$ & $\begin{array}{l}5.3^{*} \\
(1.4) \\
\end{array}$ & $\begin{array}{l}4.3^{*} \\
(1.1) \\
\end{array}$ \\
\hline & Shoulder & $\begin{array}{l}3.7^{*} \\
(1.5)\end{array}$ & $\begin{array}{l}4.4^{*} \\
(1.3)\end{array}$ & $\begin{array}{l}4.8^{*} \\
(1.1)\end{array}$ & $\begin{array}{l}4.2 * \\
(1.4)\end{array}$ & $\begin{array}{l}4.7^{*} \\
(1.5)\end{array}$ & $\begin{array}{l}3.7^{*} \\
(1.1)\end{array}$ \\
\hline $\begin{array}{c}\text { Height } \\
\text { suitability }\end{array}$ & Head & $\begin{array}{c}4.9 \\
(1.3) \\
\end{array}$ & $\begin{array}{c}5.1 \\
(1.3) \\
\end{array}$ & $\begin{array}{c}4.8 \\
(1.5) \\
\end{array}$ & $\begin{array}{c}5.5 \\
(1.1) \\
\end{array}$ & $\begin{array}{l}5.0 \\
(1.4) \\
\end{array}$ & $\begin{array}{c}5.1 \\
(1.1) \\
\end{array}$ \\
\hline \multirow[t]{2}{*}{$\begin{array}{c}\text { Shape } \\
\text { suitability }\end{array}$} & Neck & $\begin{array}{l}4.4^{*} \\
(1.3) \\
\end{array}$ & $\begin{array}{l}5.1^{*} \\
(1.3) \\
\end{array}$ & $\begin{array}{l}5.2^{*} \\
(1.3) \\
\end{array}$ & $\begin{array}{l}5.6^{*} \\
(0.9) \\
\end{array}$ & $\begin{array}{l}5.1 * \\
(1.1) \\
\end{array}$ & $\begin{array}{l}4.6^{*} \\
(1.6) \\
\end{array}$ \\
\hline & Shoulder & $\begin{array}{l}4.1^{*} \\
(1.4) \\
\end{array}$ & $\begin{array}{l}4.6^{*} \\
(1.4) \\
\end{array}$ & $\begin{array}{l}5.0^{*} \\
(1.5) \\
\end{array}$ & $\begin{array}{l}4.8^{*} \\
(1.2) \\
\end{array}$ & $\begin{array}{l}4.7^{*} \\
(1.3) \\
\end{array}$ & $\begin{array}{l}4.4^{*} \\
(1.4) \\
\end{array}$ \\
\hline \multicolumn{2}{|c|}{$\begin{array}{c}\text { Temperature } \\
\text { suitability }\end{array}$} & $\begin{array}{c}4.9 \\
(1.3)\end{array}$ & $\begin{array}{c}5.1 \\
(1.3)\end{array}$ & $\begin{array}{c}5.3 \\
(1.3)\end{array}$ & $\begin{array}{c}5.3 \\
(1.0)\end{array}$ & $\begin{array}{c}4.9 \\
(1.2)\end{array}$ & $\begin{array}{c}5.1 \\
(1.7)\end{array}$ \\
\hline \multicolumn{2}{|c|}{$\begin{array}{c}\text { Overall } \\
\text { satisfaction }\end{array}$} & $\begin{array}{c}4.7 \\
(1.3) \\
\end{array}$ & $\begin{array}{c}5.0 \\
(1.2) \\
\end{array}$ & $\begin{array}{c}5.1 \\
(1.3) \\
\end{array}$ & $\begin{array}{c}5.1 \\
(1.2) \\
\end{array}$ & $\begin{array}{c}5.1 \\
(1.5) \\
\end{array}$ & $\begin{array}{c}4.7 \\
(1.0) \\
\end{array}$ \\
\hline \multicolumn{2}{|c|}{$\begin{array}{l}\text { Willingness to } \\
\text { repurchase }\end{array}$} & $\begin{array}{c}4.3 \\
(1.7)\end{array}$ & $\begin{array}{c}4.8 \\
(1.4)\end{array}$ & $\begin{array}{c}4.8 \\
(1.7)\end{array}$ & $\begin{array}{l}5.0 \\
(1.5)\end{array}$ & $\begin{array}{l}5.0 \\
(1.7)\end{array}$ & $\begin{array}{c}4.4 \\
(1.3)\end{array}$ \\
\hline
\end{tabular}

Table 7 shows the results of multiple linear regression analysis. Less fatigue of the head and neck, less shoulder pain and comfort when changing position during sleep have significant effects on quality of sleep.

Table 7. Results of regression analysis between sleeping symptoms and sleep quality.

\begin{tabular}{ccccccc}
\hline Dependent Variable & Independent Variables: Sleeping Symptom & $\mathbf{B}$ & $\boldsymbol{\beta}$ & $\mathbf{t}$ & \multicolumn{1}{c}{$\boldsymbol{p}$-Value } \\
\hline \multirow{3}{*}{ Less Fatigue } & Head & 0.243 & 0.225 & 4.573 & 0.000 \\
\cline { 3 - 6 } & & Neck & 0.150 & 0.164 & 2.626 & 0.009 \\
\cline { 3 - 6 } & Less Pain & Shoulder & 0.094 & 0.104 & 1.816 & 0.070 \\
\cline { 2 - 6 } & & Head & 0.104 & 0.103 & 1.872 & 0.062 \\
\cline { 3 - 6 } & Neck & 0.110 & 0.122 & 1.795 & 0.074 \\
\cline { 2 - 6 } & Shoulder & 0.139 & 0.158 & 2.631 & 0.009 \\
\cline { 2 - 6 } & Comfort changing position & 0.082 & 0.090 & 1.991 & 0.047 \\
\cline { 2 - 6 } & Less Snoring or coughing & 0.023 & 0.029 & 0.560 & 0.576 \\
\cline { 2 - 6 } & Breathing & 0.025 & 0.027 & 0.485 & 0.628 \\
\hline
\end{tabular}

Table 8 shows the results of multiple linear regression analyses of sleeping positions (supine and lateral) identifying the relationship between pillow design factors and major sleeping symptoms that have significant effects on the sleep quality. The stepwise method was used to select independent variables for the regression analyses. As shown in Table 8, the significant pillow factors for major sleeping symptoms were slightly different according to sleeping positions. Overall, sleeping symptoms were significantly impacted by the corresponding pillow factors except for the shoulder area. Also, the shape factors were significant for every major symptom, but the height suitability factors were significant only for the lateral position. 
Table 8. Results of multiple linear regression analyses between major sleeping symptoms and pillow design factors according to sleeping positions.

\begin{tabular}{|c|c|c|c|c|c|c|}
\hline $\begin{array}{l}\text { Dependent Variables: Major } \\
\text { Sleeping Symptoms }\end{array}$ & Sleeping Position & Independent Variables: Pillow Factors & B & $\beta$ & $\mathbf{t}$ & $p$-Value \\
\hline \multirow{5}{*}{ Less Head Fatigue } & \multirow{3}{*}{ Supine } & Head Support & 0.382 & 0.372 & 5.738 & 0.000 \\
\hline & & Head Shape & 0.263 & 0.268 & 3.383 & 0.001 \\
\hline & & Neck Shape & 0.210 & 0.234 & 3.331 & 0.001 \\
\hline & \multirow[b]{2}{*}{ Lateral } & Head Support & 0.291 & 0.310 & 3.985 & 0.000 \\
\hline & & Head Shape & 0.430 & 0.459 & 5.910 & 0.000 \\
\hline \multirow{5}{*}{ Less Neck Fatigue } & \multirow{2}{*}{ Supine } & Neck Support & 0.236 & 0.240 & 3.565 & 0.000 \\
\hline & & Neck Shape & 0.543 & 0.520 & 7.719 & 0.000 \\
\hline & \multirow{3}{*}{ Lateral } & Neck Support & 0.249 & 0.234 & 2.934 & 0.004 \\
\hline & & Neck Height & 0.294 & 0.291 & 3.208 & 0.002 \\
\hline & & Neck Shape & 0.287 & 0.271 & 2.799 & 0.006 \\
\hline \multirow{4}{*}{ Less Shoulder Pain } & \multirow{2}{*}{ Supine } & Neck Height & 0.199 & 0.193 & 2.559 & 0.011 \\
\hline & & Shoulder Shape & 0.487 & 0.447 & 5.941 & 0.000 \\
\hline & \multirow{2}{*}{ Lateral } & Neck Height & 0.296 & 0.275 & 3.371 & 0.001 \\
\hline & & Shoulder Shape & 0.386 & 0.372 & 4.561 & 0.000 \\
\hline
\end{tabular}

Figure 1 shows the LSD post-hoc results of major sleeping symptoms that were significantly different by pillow type. There were significant differences in neck fatigue (F $(5320)=3.223$; $p$-value $=0.007)$ and shoulder pain $(\mathrm{F}(5320)=2.536 ; p$-value $=0.029)$ according to pillow type. The results of the ANOVA between the major sleeping symptoms and pillow types according to sleeping position showed no significant difference for the supine position. Otherwise, those sleeping in the lateral position reported significantly less shoulder pain $(\mathrm{F}(4138)=2.688$; $p$-value $=0.034)$. The results of post-hoc analysis using Fisher's LSD test showed that regular-type pillows scored lower than other pillow types, and peanut-shaped pillows and contour-type pillows scored higher for less symptoms than other pillow types. Overall, functional type pillows scored lower for shoulder pain. In particular, in the lateral position, the lowest scores were found in the functional B-type pillows, and the scores were significantly different from the functional A-type pillows.

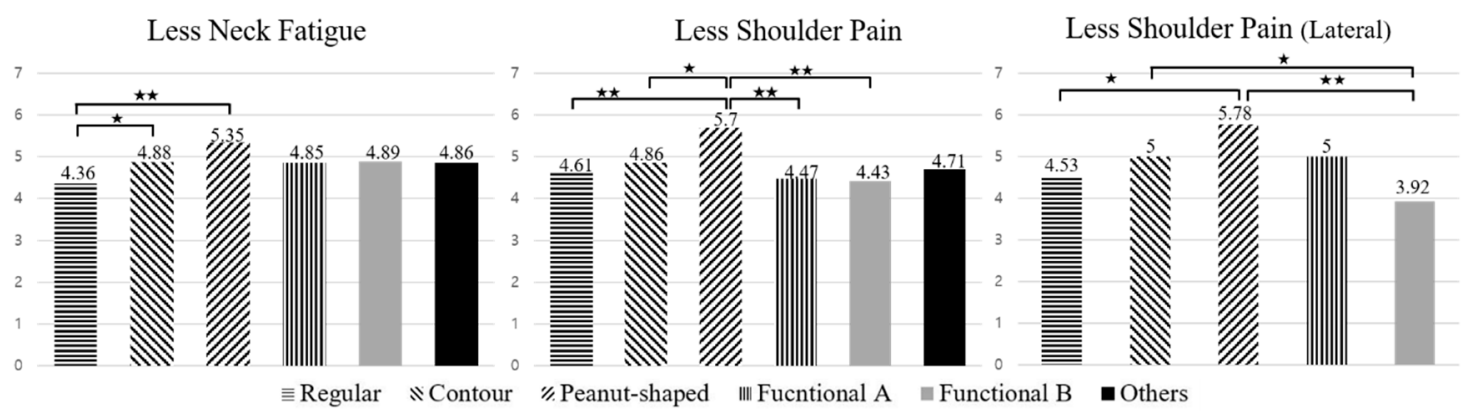

Figure 1. Post-hoc test results using Fisher's least significant difference (LSD) method for major sleeping symptoms by pillow types ( $\star \star: p<0.01, \star: p<0.05)$.

Regarding pillow materials (feathers, cotton, plastic capsules, memory foam, latex and natural material), pillows made of cotton had the highest proportion of users $(37 \% ; 121$ out of 326$)$, followed by latex and memory foam ( $23 \%$; 75 out of 326$)$, feathers $(7 \%$; 22 out of 326$)$, natural materials $(6 \% ; 21$ out of 326) and plastic capsules (4\%; 13 out of 326). Neck fatigue differed significantly by material, with plastic capsule (Mean $=5, \mathrm{SD}=1.29$ ), memory foam (Mean $=4.92, \mathrm{SD}=1.47$ ) and latex materials (Mean $=4.95, \mathrm{SD}=1.28$ ) showing less neck fatigue than other materials.

\section{Discussion}

Deyonker et al. [41] reported that the average sleep duration of healthy adults is about $7 \mathrm{~h}$, and that most adults sleep for at least $6.5 \mathrm{~h}$. The Korean National Center for Health Statistics reported in 1977 that most people sleep for more than $7 \mathrm{~h}$, but less than $8 \mathrm{~h}$ [42]. In the current study, the average sleep duration of Korean adults was $6 \mathrm{~h} 48 \mathrm{~min}$, which is less than the average sleep duration of $7 \mathrm{~h}$ 
2 min reported for British adults (mean $=7.04 \mathrm{~h}, \mathrm{SD}=1.55 \mathrm{~h}$ ) [43], and less than the recommended sleep duration of $7-8 \mathrm{~h}[16,44]$. The overall sleep quality for our participants was 4.84 points and considered slightly poor after being assessed on a seven-point scale (1 (low sleep quality) to 7 (high sleep quality)). This finding was similar to that reported by Keklund [45] for sleep quality of American adults (3.4 points on a five-point scale). The average bedtime was 12:06 a.m., and the average wake-up time was 6:54 a.m. American adults previously reported going to bed at 11:28 p.m. on average [46]. This difference could be due to the high ratio of younger to older people in the current study. In addition, the score for sleepiness during waking hours was lower (4.4 points), indicating some degree of sleepiness during the day ( 1 (discomfort) to 7 (comfort)), which suggests that daytime sleepiness is perceived as a greater discomfort than other related symptoms. Young people experienced more daytime sleepiness than middle-aged or elderly people, likely due to the excessive use of smartphones and digital media, which can interfere with sleep and cause fatigue during the day [47-50]. The proportion of young people (20s-30s) in our study population was more than $50 \%$, which could account for some degree of sleepiness reported during waking hours.

Most participants reported sleeping mainly in a supine position with some time in a lateral position, followed by participants who mainly slept in a lateral position with some time in a supine position. This may be to avoid blood stasis when maintaining the same sleeping position for too long [51]. The percentage of individuals who slept mostly or entirely in a supine position (52\%) was slightly higher than that of individuals who slept mostly or entirely in a lateral position (44\%). This is in contrast to a study of Park and Lee [11], who reported that a lateral position was most common for Korean adults. According to the results of this study, participants who usually slept in a lateral position were more unsatisfied with head support and head shape suitability of the pillow and experienced less neck support and more neck fatigue than those who slept in a supine position. In other words, the pillows the participants were using did not seem to be offering adequate support for those who slept in a lateral position. Sleeping position differed significantly by sex. The ratio of "Mainly sleeping in a supine position but also sleeping in a lateral position" was high in both males and females (males: 50\%; females: $39 \%$ ), and the ratio of "Mainly sleeping in a supine position" was low (males: $12 \%$; females: $5 \%$ ). However, when the sleeping position was divided into supine and lateral positions, the percentage of females who slept on their sides was higher than the percentage of males who slept on their sides (male: $40 \%$; female: $53 \%$ ), which is consistent with the findings of Choi et al. [52].

Participants reported tossing and turning or waking twice a night on average. This is slightly more than the 1.5 times reported by Choi et al. [53] for 1000 residents of Seoul. Among the statistically significant data observed by age groups, the natural aging phenomenon may explain the increases in the number of tossing and turning or waking events, earlier bedtime and wake-up time, breathing discomfort, snoring and coughing during sleep and morning headaches experienced among the older groups $[54,55]$. Unlike the results of this study, there were some studies that showed no significant difference by age [55], but our results were consistent with the preponderances of studies that reported a difference in those overall sleep habits and symptoms by age groups [52,56,57].

There was a significant difference between age groups in neck fatigue and shoulder support and temperature suitability of the pillow. Younger participants reported more neck and shoulder fatigue than older participants. Pain in the neck area after sleep may be due to poor posture during everyday tasks when in a static position for long periods [11], which could be why individuals in their 20s and 30s experienced more neck pain, as they spend a large amount of time staring at computer or smartphone screens. For shoulder support, older participants can overcome neck and shoulder discomfort by paying attention to physical health and investing in products that can improve neck and shoulder health.

Regarding sleep quality and symptoms, pillow shape was the main factor causing the major sleeping symptoms (head fatigue, neck fatigue and shoulder pain) that affected sleep quality. Therefore, it could be concluded that proper shape design for each part (head, neck and shoulders) is the most important factor of an effective pillow for optimal comfort. Also, according to Figure 1, overall scores 
were low for regular-type pillows and high for peanut-shaped and contour-type pillows, implying that pillows with a flat horizontal plane and gentle vertical curve for the neck and head area could be an effective shape for pillows. In cases of functional type pillows with a large height difference between the side and center area, suitability scores were different according to type of pillow. Therefore, the effectiveness of these pillow types requires further study for the validation of their effectiveness and comfort.

According to the results of our study, effective pillow characteristics are different according to sleeping position. Overall, neck shape suitability had a significant effect on head fatigue in the supine position, unlike the lateral position. This could be attributed to the difference in supporting areas between sleeping positions. When sleeping in a supine position, both the head and neck areas of a pillow support their respective area of body, but less neck support in a lateral position. For neck fatigue, height suitability was additionally significant for the lateral position. This may mean that the pillows available on the market were not properly considered for their height according to a lateral position. For shoulder pain, the significant variables were similar for different sleeping positions, but the neck-height suitability was higher for the lateral position than the supine position. This means that shoulder discomfort may be increased as a result of improper neck height in the lateral position. Therefore, there is a need to identify the adequate neck height for those who sleep mainly in a lateral position in order to reduce neck fatigue and shoulder pain.

Neck fatigue differed significantly between pillow materials and symptoms related to sleep quality. Neck fatigue was rarer among users of pillows filled with plastic capsules, latex or memory foam than among those using pillows filled with feathers or cotton. Plastic capsules, latex and memory foam are more elastic and less fluffy than feathers and cotton, allowing them to maintain their shape better and possibly offer better neck support. Therefore, a lower level of fluffiness and a higher degree of elasticity are expected to help relieve neck fatigue. Thus, to reduce sleep discomfort for a user, a pillow that supports the neck with a material such as latex or memory foam is appropriate.

This study has the following limitations. First, many of the participants in this study were university students and young adults working at Kyung Hee University, and fewer middle-aged and elderly people responded than younger people. Therefore, the reported results of this study could be biased toward the younger population. Although more than 300 people responded, the results cannot be generalized to all Korean adults. In addition, this study did not consider factors such as occupation, smoking status, coffee intake and other physical or mental illnesses that could have an impact on sleep. There were also some limitations to the subjective sleep quality questionnaire. We asked about overall sleep habits and symptoms related to sleep quality, but we did not conduct objective assessments, (e.g., estimation of sleep using actigraphs). It was also not possible to compare the sleep data directly with the PSQI, since the overall quality of sleep score in this study was derived from questions from a few questionnaires, although mostly based off the PSQI-K. In addition, we did not include the ear, an important body part with respect to comfort and pillow use when sleeping, in this study. Thus, there is a need for further research, as pain may develop with an ear leaning on the pillow. In terms of pillow shape and size, since our study did not measure the actual size of the pillows and did not collect the detailed pillow shapes, we could not provide detailed pillow design guidelines. Even in pillow materials, the plastic capsules and feathers were $6.7 \%$ and $4.0 \%$ of the total pillows, respectively, compared to $37.1 \%$ for cotton and $23 \%$ for memory foam. The balance of the number of experimental pillows remains a limitation of this study. Attention should also be paid to other pillow characteristics such as hardness and thermal conductivity. Furthermore, sleep quality can be influenced not only by pillow comfort but also by mattresses and individual lifestyle factors. Therefore, future research should be conducted to balance the sex and age of the participants, consider a variety of external variables and clarify the differences among pillow design to unravel the detailed relationship between sleep quality and pillow design factors. 


\section{Conclusions}

This study investigated sleep habits, sleeping symptoms and the comfort and support characteristics of pillows, and analyzed their relationships with each other. The overall quality score of sleep for Korean adults was on a similar level to other countries. Our participants, on average, reported a later bedtime, shorter sleep duration, more tossing and turning and waking and more daytime sleepiness than previous studies. This study reported frequent changes in sleeping positions, and the supine position was the preferred sleeping position. The sleep-related discomforts that negatively affected sleep quality were head and neck fatigue, shoulder pain and postural change during sleep. In order to reduce the negative symptoms of the head, neck and shoulders, it is important to properly design a pillow's shape. In cases of neck fatigue, it is desirable to design a pillow using materials such as latex or memory foam that have good neck support. In addition, to reduce neck fatigue and shoulder pain, it is necessary to consider the proper height of the neck support area for the lateral position. The findings of this study contribute to a better understanding of pillow comfort and support and their potential impact on sleep quality, suggesting the importance of pillow designs.

Author Contributions: All authors contributed extensively to this paper. S.J. and J.S. provided ideas and wrote the manuscript. S.B. (Sangwoo Bahn) coordinated and helped with drafting of the manuscript. J.K., H.S., and S.B. (Seonghwan Bang) implemented the literature study. All authors have read and approved the final manuscript.

Funding: This work was supported by a grant from Kyung Hee University in 2017 (KHU-20170712).

Conflicts of Interest: The authors declare no conflicts of interest.

\section{References}

1. Parsons, H.M. The bedroom. Hum. Factors 1972, 14, 421-450. [CrossRef]

2. Hartman, E.L. The Function of Sleep; Yale University Press: Oxford, UK, 1973.

3. Southwell, M.T.; Wistow, G. Sleep in hospitals at night: Are patients' needs being met? J. Adv. Nurs. 1995, 21, 1101-1109. [CrossRef] [PubMed]

4. Jung, S.H.; Park, J. Effect of life stress on the sleeping disorder of university student. J. Korea Electron Commun. Sci. 2013, 8, 345-353.

5. Pilcher, J.J.; Ginter, D.R.; Sadowsky, B. Sleep quality versus sleep quantity: Relationships between sleep and measures of health, well-being and sleepiness in college students. J. Psychosom. Res. 1997, 42, 583-596. [CrossRef]

6. Ohayon, M.; Wickwire, E.M.; Hirshkowitz, M.; Albert, S.M.; Avidan, A.; Daly, F.J.; Hazen, N. National Sleep Foundation's sleep quality recommendations: First report. Sleep Health 2017, 3, 6-19. [CrossRef] [PubMed]

7. Galinsky, A.M.; Ward, B.W.; Joestl, S.S.; Dahlhamer, J.M. Sleep duration, sleep quality, and sexual orientation: Findings from the 2013-2015 National Health Interview Survey. Sleep Health 2018, 4, 56-62. [CrossRef]

8. Exelmans, L.; Van den Bulck, J. Bedtime mobile phone use and sleep in adults. Soc. Sci. Med. 2016, 148, 93-101. [CrossRef]

9. Reyner, A.; Horne, J.A. Gender- and age-related differences in sleep determined by home-recorded sleep logs and actimetry from 400 adults. Sleep 1995, 18, 127-134.

10. Adams, R.J.; Appleton, S.L.; Taylor, A.W.; Gill, T.K.; Lang, C.; McEvoy, R.D.; Antic, N.A. Sleep health of Australian adults in 2016: Results of the 2016 Sleep Health Foundation national survey. Sleep Health 2017, 3 , 35-42. [CrossRef]

11. Park, H.J.; Lee, H.J. Survey on the Sleep time \& Posture of Korean. Available online: http://m.riss.kr/search/ detail/DetailView.do?p_mat_type=1a0202e37d52c72d\&control_no=85fce3205bbd44c8c85d2949c297615a (accessed on 15 December 2019).

12. Yang, S.H. The effect of sleep environment elements on the neck pain occurrence. J. Korea Entertain. Ind. Assoc. 2014, 8, 241-248. [CrossRef]

13. Ryu, S.Y.; Kim, K.S.; Han, M.A. Factors associated with sleep duration in Korean adults: Results of a 2008 community health survey in Gwangju metropolitan city, Korea. J. Korean Med. Sci. 2011, 26, 1124-1131. [CrossRef] [PubMed] 
14. Kim, S.J.; Lyoo, I.K.; Won, C.Y.; Jeong, D.U. Characteristics of sleep pattern among Korean college students. Sleep Med. Psychophysiol. 2006, 13, 15-21. [CrossRef]

15. Kim, S.M.; Oh, J.J.; Song, M.S.; Park, Y.H. Comparison of sleep and related variables between young and old adults. J. Korean Acad. Nurs. 1997, 27, 820-830. [CrossRef]

16. Kim, S.Y. Factors related to sleep duration in Korean adults. J. Korean Data Inf. Sci. Soc. 2018, 29, $153-165$.

17. Gordon, S.J.; Grimmer-Somers, K.A.; Trott, P.H. A randomized, comparative trial: Does pillow type alter cervico-thoracic spinal posture when side lying? J. Multidiscip. Healthc. 2011, 4, 321-327. [CrossRef]

18. Park, S.J.; Lee, H.J. Development of selection system of comfort pillow. In Proceedings of the Human Factors and Ergonomics Society Annual Meeting, Denver, CO, USA, 13 October 2003; pp. 816-820.

19. Shields, N.; Capper, J.; Polak, T.; Taylor, N. Are cervical pillows effective in reducing neck pain? N. Z. J. Physiother. 2006, 34, 3-9.

20. Gordon, S.J.; Grimmer-Somers, K.; Trott, P. Pillow use: The behavior of cervical pain, sleep quality and pillow comfort in side sleepers. Man. Ther. 2009, 14, 671-678. [CrossRef]

21. Gordon, S.J.; Grimmer-Somers, K.A.; Trott, P.H. Pillow use: The behavior of cervical stiffness, headache and scapular/arm pain. J. Pain Res. 2010, 3, 137-145.

22. Hur, J.G.; Yang, Y.A. The effect of Ergonomic pillow in patient with chronic neck pain. J. Ergon. Soc. Korea 2006, 25, 17-25.

23. Persson, L. Neck pain and pillows-A blinded study of the effect of pillows on non-specific neck pain, headache and sleep. Adv. Physiother. 2006, 8, 122-127. [CrossRef]

24. Kushida, C.A.; Sherrill, C.M.; Hong, S.C.; Palombini, L.; Hyde, P.; Dement, W.C. Cervical positioning for reduction of sleep-disordered breathing in mild-to-moderate OSAS. Sleep Breath. 2001, 5, 71-78. [CrossRef] [PubMed]

25. Cai, D.; Chen, H.L. Ergonomic approach for pillow concept design. Appl. Ergon. 2016, 52, 142-150. [CrossRef] [PubMed]

26. Ren, S.; Wong, D.W.C.; Yang, H.; Zhou, Y.; Lin, J.; Zhang, M. Effect of pillow height on the biomechanics of the head neck complex: Investigation of the cranio-cervial pressure and cervical spine alignment. PeerJ 2016, 4, e2397. [CrossRef]

27. Liu, S.F.; Lee, Y.L.; Liang, J.C. Shape design of an optimal comfortable pillow based on the analytic hierarchy process method. J. Chiropr. Med. 2011, 10, 229-239. [CrossRef] [PubMed]

28. Snyder-Halpern, R.; Verran, J.A. Instrumentation to describe subjective sleep characteristics in healthy subjects. Res. Nurs. Health 1987, 10, 155-163. [CrossRef] [PubMed]

29. Johns, M.W.; Gay, T.J.; Goodyear, M.D.; Masterton, J.P. Sleep habits of healthy young adults: Use of a sleep questionnaire. Br. J. Prev. Soc. Med. 1971, 25, 236-241. [CrossRef]

30. Johns, M.W. Factor analysis of objective and subjective characteristics of a night's sleep. Psychol. Med. 1975, 5, 413-418. [CrossRef]

31. Merica, H.; Gaillard, J.M. Statistical description and evaluation of the interrelationships of standard sleep variables for normal subjects. Sleep 1985, 8, 261-273. [CrossRef]

32. Dement, W.C.; Miles, L.E.; Carskadon, M.A. “White paper" on sleep and aging. J. Am. Geriatr. Soc. 1982, 30, 25-50. [CrossRef]

33. Buysse, D.J.; Reynolds, C.F., III; Monk, T.H.; Berman, S.R.; Kupfer, D.J. The Pittsburgh Sleep Quality Index: A new instrument for psychiatric practice and research. Psychiatry Res. 1989, 28, 193-213. [CrossRef]

34. Åkerstedt, T.; Hume, K.E.N.; minors, D.; Waterhouse, J.I.M. The subjective meaning of good sleep, an intraindividual approach using the Karolinska Sleep Diary. Percept. Motor Ski. 1994, 79, 287-296. [CrossRef] [PubMed]

35. Buysse, D.J.; Reynolds, C.F., III; Monk, T.H.; Hoch, C.C.; Yeager, A.L.; Kupfer, D.J. Quantification of subjective sleep quality in healthy elderly men and women using the Pittsburgh Sleep Quality Index (PSQI). Sleep 1991, 14, 331-338. [PubMed]

36. Carpenter, J.S.; Andrykowski, M.A. Psychometric evaluation of the Pittsburgh sleep quality index. J. Psychosom. Res. 1998, 45, 5-13. [CrossRef]

37. Aloba, O.O.; Adewuya, A.O.; Ola, B.A.; Mapayi, B.M. Validity of the Pittsburgh sleep quality index (PSQI) among Nigerian university students. Sleep Med. 2007, 8, 266-270. [CrossRef]

38. Sohn, S.I.; Kim, D.H.; Lee, M.Y.; Cho, Y.W. The reliability and validity of the Korean version of the Pittsburgh Sleep Quality Index. Sleep Breath. 2012, 16, 803-812. [CrossRef] [PubMed] 
39. Yokura, H.; Nakanishi, M.; Niwa, M. Using the compression properties of pillows to estimate sleeping comfort. Int. J. Cloth. Sci. Technol. 1999, 11, 160-169. [CrossRef]

40. WHO. The Asia-Pacific Perspective: Redefining Obesity and Its Treatment; Health Communications: Sydney, Australia, 2000.

41. Deyonker, O.; Zee, P.; Reid, K.; Attarian, H.; Wolfe, L.; Baron, K.G. 0808 Social jet lag; Association with sleep duration and quality among healthy adults. Sleep 2017, 40, A299. [CrossRef]

42. Lamberg, L. The American Medical Association Guide to Better Sleep; Random House Inc.: New York, NY, USA, $1984 ; 342 p$.

43. Groeger, J.A.; Zijlstra, F.R.H.; Dijk, D.J. Sleep quantity, sleep difficulties and their perceived consequences in a representative sample of some 2000 British adults. J. Sleep Res. 2004, 13, 359-371. [CrossRef]

44. Hirshkowitz, M.; Whiton, K.; Albert, S.M.; Alessi, C.; Bruni, O.; DonCarlos, L.; Kheirandish-Gozal, L. National Sleep Foundation's updated sleep duration recommendations. Sleep Health 2015, 1, 233-243. [CrossRef]

45. Keklund, G.; ÅKerstedt, T. Objective components of individual differences in subjective sleep quality. J. Sleep Res. 1997, 6, 217-220. [CrossRef]

46. Carrier, J.; Monk, T.H.; Buysse, D.J.; Kupfer, D.J. Sleep and morningness-eveningness in the 'middle'years of life (20-59y). J. Sleep Res. 1997, 6, 230-237. [CrossRef] [PubMed]

47. Liu, X.; Uchiyama, M.; Kim, K.; Okawa, M.; Shibui, K.; Kudo, Y.; Doi, Y.; minowa, M.; Ogihara, R. Sleep loss and daytime sleepiness in the general adult population of Japan. Psychiatry Res. 2000, 93, 1-11. [CrossRef]

48. Jung, M.S.; Ju, K.O.; Song, M.S.; Lee, K.S. Effects of Smartphone Overuse on Perceived Cognitive Function, Fatigue, and Daytime Sleepiness among College Students. J. Korean Soc. Sch. Health 2016, 29, 245-255. [CrossRef]

49. Kim, S.J. The present and prospect of Online Video, Music service and Media Usage. J. Digit. Contents Soc. 2015, 16, 137-144. [CrossRef]

50. Zarghami, M.; Khalilian, A.; Setareh, J.; Salehpour, G. The impact of using cell phones after light-out on sleep quality, headache, tiredness, and distractibility among students of a university in North of Iran. Iran. J. Psychiatry Behav. Sci. 2015, 9, e2010. [CrossRef]

51. Agrawal, K.; Chauhan, N. Pressure ulcers: Back to the basic. Indian J. Plast. Surg. Off. Publ. Assoc. Plast. Surg. India 2012, 45, 244. [CrossRef]

52. Choi, J.Y.; Koo, W.M.; Song, C.G.; Lee, J.H.; Lim, J.O.; Kwon, S.A. The use and satisfaction of pillow by gender and age. J. Hum. Ecol. 2008, 12, 251-265.

53. Choi, S.H.; Kim, R.; Suh, K.Y. A Study on the Sleep Patterns of the General Adult Population in Seoul. J. Korean Neuropsychiatr. Assoc. 1992, 31, 289-309.

54. Kim, T.; Jeong, H.G.; Im, J.E.; Jo, G.M.; Park, S.H.; Lee, G.W.; Kim, S.H. Snoring Prevalence between Male and Female According to Aging: Preliminary Study Exploring a Risk Factor of Menopause. J. Menopausal Med. 2003, 9, 149-155.

55. Mirer, A.G.; Young, T.; Palta, M.; Benca, R.M.; Rasmuson, A.; Peppard, P.M. Sleep-disordered breathing and the menopausal transition among participants in the Sleep in Midlife Women Study. Menopause 2017, $24,157$. [CrossRef]

56. Hayashi, Y.; Endo, S. AU-Night sleep polygraphic recordings of healthy aged persons: REM and Slow-Wave sleep. Sleep 1982, 5, 277-283. [CrossRef] [PubMed]

57. Clapin-French, E. Sleep patterns of aged persons in long-term care facilities. J. Adv. Nurs. 1986, 11, 57-66. [CrossRef] [PubMed]

(C) 2020 by the authors. Licensee MDPI, Basel, Switzerland. This article is an open access article distributed under the terms and conditions of the Creative Commons Attribution (CC BY) license (http://creativecommons.org/licenses/by/4.0/). 